Алгебра и анализ

Toм 21 (2009), № 2
St. Petersburg Math. J.

Vol. 21 (2010), No. 2, Pages 309-315

S 1061-0022(10)01096-4

Article electronically published on January 26, 2010

\title{
DUALITY THEOREM FOR MOTIVES
}

\author{
I. A. PANIN AND S. A. YAGUNOV
}

\begin{abstract}
A general duality theorem for the category of motives is established, with a short, simple, and self-contained proof.
\end{abstract}

\section{INTRODUCTION}

Recently, due to the active study of cohomological invariants in algebraic geometry, "transplantation" of classical topological constructions to the algebraic-geometrical "soil" seems to be rather important. In particular, it is very interesting to study topological properties of the category of motives.

The concept of a motive was introduced by Alexander Grothendieck in 1964 in order to formalize the notion of universal (co-)homology theory (see the detailed exposition of Grothendieck's ideas in [5]). For us, the principal example of this type is the category of motives $\mathbf{D M}^{-}$, constructed by Voevodsky 12 for algebraic varieties.

The Poincaré duality is a classical and fundamental result in algebraic topology that initially appeared in Poincaré's first topological memoir "Analysis Situs" 9] (as a part of the Betti numbers symmetry theorem proof). The proof of the general duality theorem for extraordinary cohomology theories apparently belongs to Adams [1].

Our purpose in this paper is to establish a general duality theorem for the category of motives. Essentially, we extend the main statement of $[\underline{8}$ to this category. Many known results can easily be interpreted in these terms. In particular, we get a generalization of the Friedlander-Voevodsky duality theorem [4] to the case of the ground field of arbitrary characteristic. The proof of this fact, involving the main result of [8], was kindly conveyed to the authors by Andrei Suslin in a private communication.

Being inspired by his work and Dold-Puppe's category approach [2] to the duality phenomenon in topology, we decided to present a short, simple, and self-contained proof of a similar result for the category of motives.

Our result might be viewed as a purely abstract theorem and rewritten in the spirit of "abstract nonsense" as a statement about some category with a distinguished class of morphisms. Essentially, what is required for the proof is the existence of finite fiber products and the terminal object in the category of varieties, a small part of the tensor triangulated category structure for motives, and finally, the existence of transfers for the class of morphisms generated by graphs of a special type (of projective morphisms).

However, rather, we preferred to formulate all statements for motives of algebraic varieties in order to clarify the geometric nature of the construction and make possible applications easier. This led, in particular, to the appearence of the second (co)homology index responsible for twist with the Tate object $\mathbb{Z}(1)$ (see Voevodsky [12]). The only exception is the classical Example 2,

2000 Mathematics Subject Classification. Primary 14F42.

Key words and phrases. Category of motives, Poincaré duality, smooth algebraic varieties.

Both authors are deeply grateful to SFB-701 for its financial support during their work. 


\section{§1. AXIOMS AND EXAMPLES}

Consider a covariant functor $\mathbf{M}: \mathbf{S m} / \mathbf{k} \rightarrow \mathfrak{M}$ from the category of smooth algebraic varieties over a field $\mathbf{k}$ to a tensor triangulated category $11 \mathfrak{M}$ that sends Cartesian products to tensor products.

For the terminal object $\mathrm{pt}=\mathrm{Spec} \mathbf{k}$ of the category $\mathbf{S m} / \mathbf{k}$, we denote the object $M(\mathrm{pt}) \in \mathrm{Ob} \mathfrak{M}$ by $\mathbb{Z}$. From now on, we shall often use implicitly the canonical isomorphisms

$$
\mathbb{Z} \otimes M(X) \simeq M(X) \simeq M(X) \otimes \mathbb{Z}
$$

induced by the natural identifications pt $\times X=X=X \times \mathrm{pt}$ in $\mathbf{S m} / \mathbf{k}$.

Also, we assume that the category $\mathfrak{M}$ is endowed with a fixed invertible object $\mathbb{Z}(1)$, called the Tate object. We denote the $n$-fold product $\mathbb{Z}(1) \otimes \cdots \otimes \mathbb{Z}(1)$ by $\mathbb{Z}(n)$ and $M \otimes \mathbb{Z}(n)$ by $M(n)$.

For a variety $X$, we call the object $M(X)$ in the category $\mathfrak{M}$ the (orientable) motive of $X$ and the functor $\mathbf{M}$ itself the (orientable) theory of motives on the category $\mathbf{S m} / \mathbf{k}$, provided that the axioms below are fulfilled.

- Cancellation axiom. For every integer $q$ and arbitrary varieties $X$ and $Y$ in $\mathbf{S m} / \mathbf{k}$ there exists a canonical isomorphism

$$
\operatorname{Hom}_{\mathfrak{M}}(M(X), M(Y)) \simeq \operatorname{Hom}_{\mathfrak{M}}(M(X)(q), M(Y)(q)) .
$$

- Transfer axiom. Every projective equidimensional morphism $f: X \rightarrow Y$ of codimension $d=\operatorname{dim} Y-\operatorname{dim} X$ determines a map of motives

$$
f^{!}: M(Y) \rightarrow M(X)(d)[2 d]
$$

that is functorial with respect to the specified class of morphisms, i.e., $f^{!}$(id) $=$id and $(f g)^{!}=g^{!} f$.

- Base-change axiom. For every transversal square (see, e.g., 8, Definition A.1]):

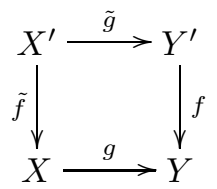

with projective equidimensional morphisms $f$ and $\tilde{f}$, the relation $2 \tilde{g}_{*} \tilde{f}^{!}=f^{!} g_{*}$ is fulfilled in $\mathfrak{M}$.

- Consistency axiom. Let $F: X \times Z \rightarrow Y \times Z$ be the morphism $f \times$ id $\in$ Mor $\mathfrak{M}$ for some $f: X \rightarrow Y$ for which the transfer is well defined. Then, the relation $F^{!}=f^{!} \otimes 1$ is fulfilled in the category $\mathfrak{M}$ for all such $f$ and $F$.

For a given theory of motives $\mathbf{M}$, we define homology and cohomology groups in the following way. Set

$$
\operatorname{HM}^{n m}(X):=\operatorname{Hom}_{\mathfrak{M}}(M(X), \mathbb{Z}(m)[n])
$$

and

$$
\operatorname{HM}_{n m}(X):=\operatorname{Hom}_{\mathfrak{M}}(\mathbb{Z}(m)[n], M(X)) .
$$

For us, the most important example of the construction described above is Voevodsky's motives.

\footnotetext{
${ }^{1}$ In particular, we assume that the tensor structure is compatible with the shift functor in such a way that $A[q] \otimes B=A \otimes B[q]$. A full list of axioms for the triangulated category can be found in [6] [7].

${ }^{2}$ Here and below $g_{*}$ et cetera denote morphisms obtained from the induced morphism $M(g)$ in the category $\mathfrak{M}$ after an application of a necessary shift and the Tate twist.
} 
Example 1. Put $\mathfrak{M}=\mathbf{D M}^{-}(\mathbf{k})$, and let the functor $\mathbf{M}$ be the corresponding motive functor as in [10, 12. Then $\mathbf{M}$ happens to be a functor with transfers for projective morphisms and our axioms are fulfilled. The cancellation axiom was checked in this context in [11. Construction of the transfer and verification of other axioms belong to the basic properties of Voevodsky's motives (see, e.g., [10, §4]).

Example 2. Changing, in our settings, $\mathbf{S m} / \mathbf{k}$ with a category of smooth topological varieties and taking the derived category of $\mathbb{Z}_{2}$-modules as $\mathfrak{M}$, we obtain, as can easily be seen, the standard definitions of singular (co-)homology. To make the indexes consistent, it suffices to put $M(i)[j]:=M[j-i]$, where on the right-hand side the usual triangulated category shift is employed. Alliteratively, one may simply rewrite all the constructions with a single index. The role of projective morphisms is played in this case by proper differentiable maps. The transfer construction can be found in almost any algebraic topology textbook (see, e.g., [3]). Direct verification shows that all the axioms are fulfilled in the case under consideration. As a result we obtain a proof of the classical Poincaré duality theorem in the spirit of Dold-Puppe's paper [2].

\section{§2. STATEMENTS AND PROOFS}

Our purpose in the current paper is to prove the duality theorem for orientable motives. Namely, we establish the following statement.

Theorem 3. For every orientable theory of motives $\mathbf{M}$ and arbitrary varieties $X, Y \in$ $\mathbf{S m} / \mathbf{k}$ with projective equidimensional $X$, there is a canonical isomorphism of Abelian groups

$$
\operatorname{Hom}(M(Y)(i)[j], M(X)) \simeq \operatorname{Hom}(M(Y) \otimes M(X), \mathbb{Z}(d-i)[2 d-j])
$$

contravariant with respect to $Y$. Here $d=\operatorname{dim} X$.

As a simple consequence of our statement, we get the following version of the classical Poincaré duality theorem.

Corollary 4. For an arbitrary smooth projective equidimensional variety $X$ of dimension $d$, we have the canonical isomorphism

$$
\mathrm{HM}_{*, *}(X) \simeq \mathrm{HM}^{2 d-*, d-*}(X) .
$$

Proof. Set $Y=\mathrm{pt}$ in the theorem.

Within the next paragraph we assume that the category $\mathfrak{M}$ under consideration admits inner $\underline{H o m}$-objects; i.e., for every variety $X \in \mathbf{S m} / \mathbf{k}$ the tensor product functor

$$
M(X) \otimes-: \mathfrak{M} \rightarrow \mathfrak{M}
$$

has a right adjoint.

For a variety $X$ satisfying the hypothesis of Corollary 4 , we consider the canonical morphism

$$
M(X) \otimes M(X)=M(X \times X) \stackrel{\Delta^{!}}{\longrightarrow} M(X)(d)[2 d] \stackrel{p_{*}}{\longrightarrow} \mathbb{Z}(d)[2 d]
$$

and call it the motivic cofundamental class of $X$ (see the details below). This morphism determines the canonical duality map

$$
M(X) \rightarrow \underline{\operatorname{Hom}}(M(X), \mathbb{Z}(d)[2 d]) .
$$

Then the following statement is true. 
Corollary 5. Let $\mathbf{M}$ be the theory of motives considered above in Example 1. Then for every smooth projective equidimensional variety $X$ the duality morphism

$$
\psi: M(X) \rightarrow \underline{\operatorname{Hom}}(M(X), \mathbb{Z}(d)[2 d])
$$

constructed above is an isomorphism.

Proof. We complete the morphism $\psi$ up to a distinguished triangle in the category $\mathfrak{M}$ and denote the third vertex of the triangle by $\operatorname{Cone}(\psi)$. By Theorem 3 , we have $\operatorname{Hom}(M(Y)(p)[q]$, Cone $(\psi))=0$ for every smooth irreducible variety $Y$ and arbitrary integers $p, q$. Since the category $\mathfrak{M}$ is weakly generated by motives of this kind, we see that the object $\operatorname{Cone}(\psi)$ is isomorphic to the zero object. Triangulated category axioms easily imply that a morphism with zero cone is an isomorphism.

Proof of Theorem 3. In order to construct the isomorphisms mentioned in the theorem, we need the following ingredients. First, denote by $1 \in \mathrm{HM}^{0,0}(\mathrm{pt})=\mathrm{HM}_{0,0}(\mathrm{pt})$ the element of the (co-)homology group of the point that belongs to the (co-)homology group of the point and corresponds to the identity morphism id: pt $\rightarrow$ pt. Note that for a morphism of varieties $f: X \rightarrow Y$ the map $M(f)$ induces the natural maps $f_{*}: \mathrm{HM}_{*, *}(X) \rightarrow \mathrm{HM}_{*, *}(Y)$ in motivic homology and $f^{*}: \mathrm{HM}^{*, *}(Y) \rightarrow \mathrm{HM}^{*, *}(X)$ in cohomology, respectively. In the case where $f$ is projective of codimension $d$, the motivic transfer map $f^{!}$induces the corresponding transfers

$$
f_{!}: \mathrm{HM}^{*, *}(X) \rightarrow \mathrm{HM}^{*+2 d, *+d}(Y) \text { and } f^{!}: \mathrm{HM}_{*, *}(Y) \rightarrow \mathrm{HM}_{*-2 d, *-d}(X)
$$

in (co-)homology. For a variety $X$ of dimension $d$, we consider the diagonal and projection morphisms

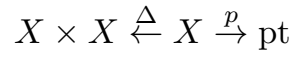

and call the elements

$$
[X]_{*}=\Delta_{*} p^{!}(1) \in \mathrm{HM}_{2 d, d}(X \times X)
$$

and

$$
[X]^{*}=\Delta_{!} p^{*}(1) \in \mathrm{HM}^{2 d, d}(X \times X)
$$

the fundamental and the cofundamental class of $X$, respectively. Also, we define the slant products

$$
/: \mathrm{HM}^{i, j}(X \times Y) \otimes \mathrm{HM}_{m, n}(Y) \rightarrow \operatorname{HM}^{i-m, j-n}(X)
$$

and

$$
\backslash: \mathrm{HM}_{m, n}(X \times Y) \otimes \mathrm{HM}^{i, j}(Y) \rightarrow \mathrm{HM}_{m-i, n-j}(X)
$$

in the following way. For elements $\alpha \in \mathrm{HM}^{i, j}(X \times Y)$ and $a \in \mathrm{HM}_{m, n}(Y)$, set

$$
\alpha / a: M(X)(m)=M(X) \otimes \mathbb{Z}(m) \stackrel{1 \otimes a}{\longrightarrow} M(X) \otimes M(Y)[-n] \stackrel{\alpha}{\longrightarrow} \mathbb{Z}(i)[j-n]
$$

for the first product, and symmetrically,

$$
\beta \backslash b: \mathbb{Z}(m)[n-j] \stackrel{b}{\longrightarrow} M(X) \otimes M(Y)[-j] \stackrel{1 \otimes \beta}{\longrightarrow} M(X) \otimes \mathbb{Z}(i)=M(X)(i)
$$

for the second, provided that $b \in \mathrm{HM}_{m, n}(X \times Y)$ and $\beta \in \mathrm{HM}^{i, j}(Y)$. (Here and below we implicitly use the cancellation axiom.) We introduce the Poincaré duality homomorphisms for the case of Corollary 4, by letting

$$
\mathcal{D} \cdot(-)=[X]^{*} /-: \operatorname{HM}_{*, *}(X) \rightarrow \operatorname{HM}^{2 d-*, d-*}(X)
$$

and

$$
\mathcal{D}^{\bullet}(-)=-\backslash[X]_{*}: \mathrm{HM}^{*, *}(X) \rightarrow \mathrm{HM}_{2 d-*, d-*}(X),
$$

verbatim as was done in 8 . 
Then, we consider a natural extension of these homomorphisms to the general case of the theorem. For brevity, denote the motive $M(Y)(i)[j]$ by $\mathcal{Y}$, and the index shift and twist $(d)[2 d]$ by $\{d\}$. We construct a map

$$
\mathcal{D}_{\bullet}: \operatorname{Hom}(\mathcal{Y},-) \rightarrow \operatorname{Hom}(\mathcal{Y} \otimes-, \mathbb{Z}\{d\})
$$

as follows. For a morphism $a \in \operatorname{Hom}(\mathcal{Y}, M(X))$, let $\mathcal{D} \cdot(a)$ be defined by the formula

$$
\mathcal{Y} \otimes M(X) \stackrel{a \otimes 1}{\longrightarrow} M(X) \otimes M(X) \stackrel{\Delta^{!}}{\longrightarrow} M(X)\{d\} \stackrel{p_{*}}{\longrightarrow} \mathbb{Z}\{d\} .
$$

The inverse map

$$
\mathcal{D}^{\bullet}: \operatorname{Hom}(\mathcal{Y} \otimes-, \mathbb{Z}\{d\}) \rightarrow \operatorname{Hom}(\mathcal{Y},-)
$$

is given for a morphism $\alpha \in \operatorname{Hom}(\mathcal{Y} \otimes M(X), \mathbb{Z}\{d\})$ as follows:

$$
\mathcal{Y} \stackrel{1 \otimes p^{!}}{\longrightarrow} \mathcal{Y} \otimes M(X)\{-d\} \stackrel{1 \otimes \Delta_{*}}{\longrightarrow} \mathcal{Y} \otimes M(X) \otimes M(X)\{-d\} \stackrel{\alpha \otimes 1}{\longrightarrow} \mathbb{Z} \otimes M(X) .
$$

Obviously, the resulting maps are contravariant with respect to the variable $\mathcal{Y}$. Now, Theorem 3 results from the cancellation axiom and the commutativity of the next two diagrams 3

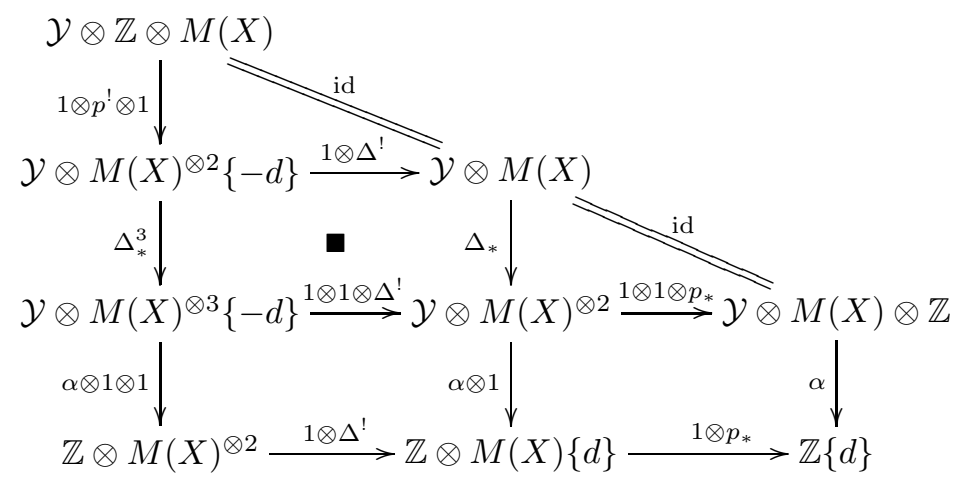

and

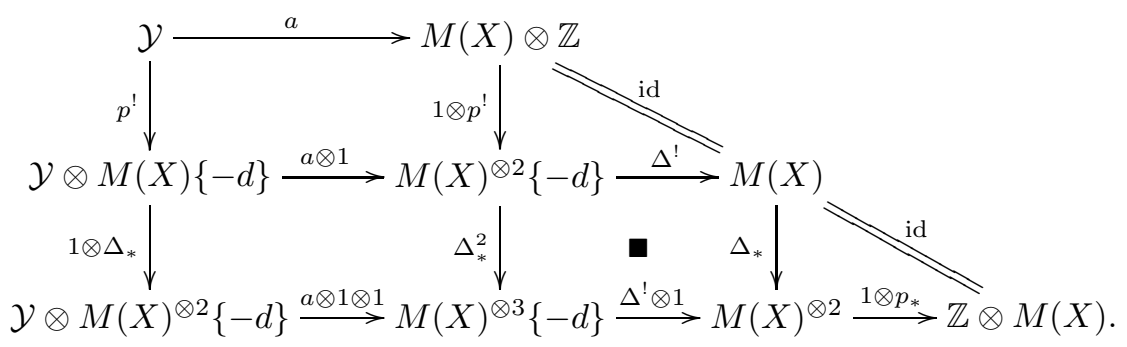

The squares marked by in both diagrams correspond to the following Cartesian transversal diagram of varieties:

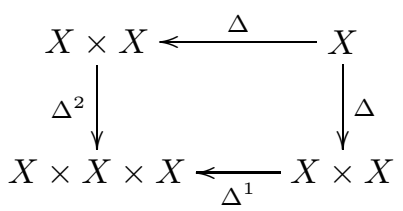

Therefore, these squares are commutative in view of the base change and consistency axioms.

\footnotetext{
${ }^{3}$ In the subsequent diagrams, $M(X)^{\otimes n}$ denotes the $n$-fold tensor product and $\Delta^{i}$ is the diagonal morphism applied to the $i$ th factor.
} 
Finally, we note that, as soon as the Poincaré duality isomorphisms have been fixed, the transfer maps in (co-)homology can be recovered uniquely, much in the same way as in the classical algebraic topology. Namely, the following is true.

Proposition 6. For projective equidimensional varieties $X, Y \in \mathbf{S m} / \mathbf{k}$ and a morphism $f: X \rightarrow Y$, we have:

$$
f_{!}=\mathcal{D}_{\bullet}^{Y} f_{*} \mathcal{D}_{X}^{\bullet} \quad \text { and } \quad f^{!}=\mathcal{D}_{X}^{\bullet} f^{*} \mathcal{D}_{\bullet}^{Y} .
$$

Here $\mathcal{D}_{\bullet}^{X}$ and $\mathcal{D}_{\bullet}^{Y}$ denote the Poincaré duality isomorphisms from Corollary 4 applied to the varieties $X$ and $Y$, respectively.

Proof. The first identity can easily be derived from the relation $f_{*}\left(\alpha \backslash[X]_{*}\right)=f_{!}(\alpha) \backslash[Y]_{*}$, which, in its turn, follows from the commutativity of the diagram

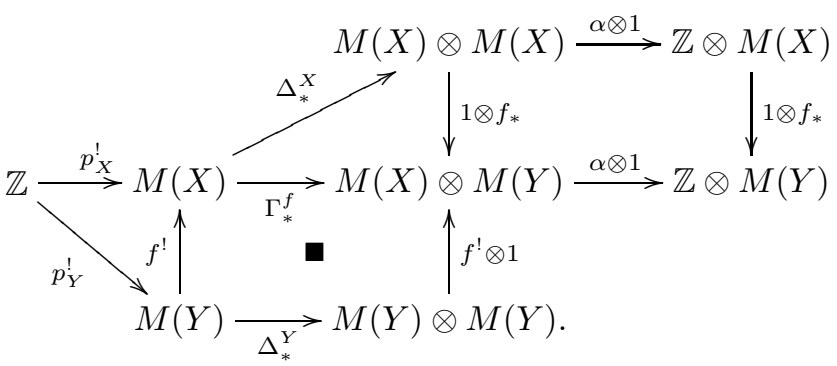

(Here all the shifts and twists are omitted for simplicity.) The square marked by $\mathbf{\square}$ is induced by the transversal graph diagram of the morphism $f$ :

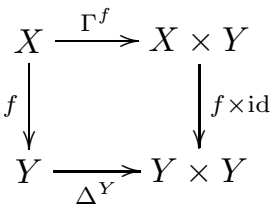

Hence, it is commutative. The commutativity in all other diagram parts is obvious. The second identity can be proved in a similar way.

\section{REFERENCES}

[1] J. F. Adams, Stable homotopy and generalized homology, Univ. of Chicago Press, Chicago-London, 1974. MR0402720 (53:6534)

[2] A. Dold and D. Puppe, Duality, trace, and transfer, Proceedings of the International Conference on Geometric Topology (Warsaw, 1978), PWN, Warsaw, 1980, pp. 81-102. MR0656721|(83f:55005)

[3] A. Fomenko and D. Fuks, A course in homotopic topology, Nauka, Moscow, 1989. (Russian) MR.1027592 (92a:55001)

[4] E. Friedlander and V. Voevodsky, Bivariant cycle cohomology, Cycles, Transfers, and Motivic Homology Theories, Ann. of Math. Stud., vol. 143, Princeton Univ. Press, Princeton, NJ, 2000, pp. 138-187. MR1764201

[5] Yu. I. Manin, Correspondences, motifs and monoidal transformations, Mat. Sb. 77 (119) (1968), no. 4, 475-507; English transl., Math. USSR-Sb. 6 (1968), 439-470. MR0258836 (41:3482)

[6] J. P. May, The additivity of traces in triangulated categories, Adv. Math. 163 (2001), no. 1, 34-73. MR 1867203 (2002k:18019)

[7] C. Mazza, V. Voevodsky, and C. Weibel, Lecture notes on motivic cohomology, Clay Math. Monogr., vol. 2, Amer. Math. Soc., Providence, RI; Clay Math. Inst., Cambridge, MA, 2006. MR2242284 (2007e:14035)

[8] I. Panin and S. Yagunov, T-spectra and Poincaré duality, J. Reine Angew. Math. 617 (2008), 193-213. MR2400995 (2009b:14039)

[9] H. Poincaré, Analysis situs, J. École Polytech. 1 (1895), 1-121. 
[10] A. Suslin and V. Voevodsky, Bloch-Kato conjecture and motivic cohomology with finite coefficients, The Arithmetic and Geometry of Algebraic Cycles (Banff, AB, 1998), NATO Sci. Ser. C Math. Phys. Sci., vol. 548, Kluwer Acad. Publ., Dordrecht, 2000, pp. 117-189. MR.1744945 (2001g:14031)

[11] V. Voevodsky, Motivic cohomology groups are isomorphic to higher Chow groups in any characteristic, Int. Math. Res. Not. 2002, no. 7, 351-355. MR1883180 (2003c:14021)

[12] - Triangulated categories of motives over a field, Cycles, Transfers, and Motivic Homology Theories, Ann. of Math. Stud., vol. 143, Princeton Univ. Press, Princeton, NJ, 2000, pp. 188-238. MR 1764202

St. Petersburg Branch, Steklov Institute of Mathematics, Russian Academy of Sciences, Fontanka 27, St. Petersburg 191023, Russia

Current address: Fakultät für Mathematik, Universität Bielefeld, Universitätstrasse, 25, Bielefeld 33615, Germany

E-mail address: panin@pdmi.ras.ru

St. Petersburg Branch, Steklov Institute of Mathematics, Russian Academy of Sciences, Fontanka 27, St. Petersburg 191023, Russia

Current address: Fakultät für Mathematik, Universität Bielefeld, Universitätstrasse, 25, Bielefeld 33615 , Germany

E-mail address: yagunov@gmail.com

Received 25/SEP/2008

Translated by THE AUTHORS 